\title{
El Paro nacional del 2021 en Colombia: estallido social entre dinámicas estructurales y de coyuntura. La relevancia de la acción política y del diálogo en su desarrollo y transformación ${ }^{1}$
}

\section{The National Strike of 2021 in Colombia: a Social Outburst Framed within Structural and Situational Dynamics. The Relevance of Political Action and Dialogue in its Development and Transformation}

\author{
Adolfo Adrián Álvarez-Rodríguez ${ }^{2}$ \\ Economista. Experto en gestión de Paz y Conflicto \\ Universidad del Valle. Cali, Colombia \\ https:/ / orcid.org/0000-0003-4884-8097• adolfo.alvarez@correounivalle.edu.co
}

\section{Presentación}

Aquí se presenta una lectura sintética del Paro Nacional ${ }^{3}$, en particular en el contexto de Cali, en clave de análisis de conflicto y su transformación, abordando los procesos de diálogo, mediación y negociación, que han conducido a un trámite parcial de esta protesta, que por su alcance en la ciudad adquirió la connotación de "estallido social" 4 .

Se parte de reconocer que se ha producido una avalancha de documentos, artículos, columnas de prensa, textos audiovisuales y mensajes, muchos escritos al calor de los acontecimientos, dirigidos a "dar cuenta" o señalar "la verdadera naturaleza" de lo acontecido en estos meses, específicamente en Cali. Algunos orientados a demostrar las "causas estructurales profundas del Paro y del Estallido" y su "justeza" e incluso a comprender todos los repertorios expresados en el contexto de esta movilización ${ }^{5}$ y a denunciar la "respuesta represiva del Estado". También constatar que se produjeron documentos críticos, centrados en mostrar las razones, actores y motivaciones detrás del "desbordamiento" y los "repertorios violentos" o incluso, del lado oficial, en señalar sólo "conspiraciones" y "motivaciones políticas".

1 Este texto es un resumen de un Artículo más amplio en preparación como parte de un Texto de Análisis sobre el Paro en particular en Cali producido a partir de la experiencia de observación, acompañamiento de algunos procesos y análisis realizada desde el Instituto de Investigación e Intervención para la paz de la Universidad del Valle y en el diálogo e interacción con colegas y personas cercanas a escenarios y procesos relacionados con ese momento.

2 Director del Instituto de Investigación e Intervención para La Paz de la Universidad del Valle, Cali, Colombia.

3 Nos referimos al "Paro Nacional" en Colombia convocado en el mes de marzo de 2021 y desarrollado entre el 28 de abril y los meses de mayo y junio, por una alianza de organizaciones: Central Unitaria Trabajadores (CUT); la Confederación Nacional del Trabajo (CGT); la Confederación de Trabajadores de Colombia (CTC); la Confederación de Pensionados de Colombia (CPC); la Confederación Democrática de los Pensionados (CDP); la Federación Colombiana de Trabajadores de la Educación (FECODE); Dignidad Agropecuaria, y la Cruzada Camionera. También, autoridades étnicas recogidas en el CRIC y plataformas políticas como Congreso de los pueblos; gremiales diversos (estudiantes, pensionados), campesinas (Dignidad Agropecuaria) y otros sectores.

4 Al respecto es interesante la reflexión de Salazar-Trujillo (2021), quien señala la dificultad en nombrarlo, no por la ausencia de términos, sino por la abundancia: explosión social, estallido social, revuelta popular, revuelta urbana, movilización, revolución, paro nacional o simplemente paro).

5 Merece referencia la publicación de la Facultad de Economía de la Universidad del Valle (Centro de Investigación y Documentación Socioeconómica [CIDSE], 2021), en la cual se analizan diferentes aspectos y cada uno puede entenderse como una lectura parcial y también articulada, sobre factores estructurales, culturales e incluso históricos y de contexto, de la dinámica del paro.

Recibido: 15/12/2021 | Aprobado: 28/12/2021 | Publicado: 01/01/2022

(i)(-) Esta obra está bajo una Licencia Creative Commons Atribución-NoComercial-CompartirIgual 4.0 Internacional.

¿Cómo citar este artículo? / How to quote this article?

Álvarez-Rodríguez, A. A. (2022). El Paro nacional del 2021 en Colombia: estallido social entre dinámicas estructurales y de coyuntura. La relevancia de la acción política y del diálogo en su desarrollo y transformación. Prospectiva. Revista de Trabajo Social e intervención social, (33), 1-12. doi: 10.25100/ prts.v0i33.11864. 
Álvarez-Rodríguez

No obstante, a ocho meses de su desenlace y si bien se ha ido decantando la discusión, aún son muchas las preguntas que se plantean en una lectura analítica comprensiva no sólo para entender las "causas" y factores que han incidido o determinado esta "explosión", sino su dinámica y los retos, dada la amenaza y la posibilidad de una reproducción. En el presente texto proponemos entonces el análisis centrado en esa dinámica y los retos en la perspectiva de entender las posibilidades y dificultades de su transformación constructiva.

\section{Introducción}

El Paro Nacional iniciado el 28 de abril del 2021, que se prolongó al menos por dos meses en el que se expresaron y se fueron sumando actores y demandas diversas a lo largo y ancho del territorio nacional, tuvo como detonante el rechazo a proyectos y políticas del Gobierno encabezado por el presidente Duque, del partido Centro Democrático y los partidos de la coalición gobernante: las reformas tributaria y a la salud, al régimen de contratación laboral y pensional. De otra parte, con demandas sobre: una renta básica, equivalente a 1 Salario mínimo mensual, la matrícula cero para el ingreso a universidades, la revisión de los TLCs y el cumplimiento de acuerdos (suscritos con estudiantes universitarios, grupos de productores del campo, indígenas y comunidades campesinas y camioneros), y respecto a los Acuerdos de Paz, su pleno cumplimiento y la no fumigación de cultivos ilícitos; la eliminación del ESMAD (Escuadrón Móvil Antidisturbios) entre otros. Si bien el Paro en un primer momento enfrentó a organizaciones sociales de carácter nacional, con el Gobierno Duque y sus políticas, amplió su alcance y abrió espacio a la expresión de conflictividades diversas con la presencia de actores más locales y la inclusión de demandas tanto al gobierno nacional como a gobiernos territoriales, como en el caso específico de Cali y el Valle del Cauca.

Se puede caracterizar como una movilización y protesta masiva, fundamentalmente pacífica ${ }^{6}$, la más grande en varios decenios, tanto a nivel nacional, como local y regional, que puso en evidencia la inconformidad de amplios sectores sociales populares y medios de Colombia, el cansancio y la angustia frente a problemáticas acumuladas -de precarización económica y desigualdades- que la pandemia y su manejo mostró y amplificó, con especial fuerza en la ciudad de Cali. No obstante, en la dinámica del Paro y especialmente en el contexto de diversos hechos de confrontación, la respuesta por parte de las autoridades policiales fue violenta. Como consecuencia, en las primeras 6 semanas se produjo cerca de medio centenar muertos (Comisión Interamericana de Derechos Humanos [CIDH], 2021, pp. 6-7), la mayoría de ellos jóvenes manifestantes y dos agentes del Estado, y múltiples violaciones de derechos humanos que alimentaron la sucesiva confrontación, y sus banderas, con la demanda de desmonte del Escuadrón Móvil Antidisturbios (ESMAD) de la Policía y la solicitud de la presencia de la Comisión Interamericana de Derechos Humanos (CIDH)7.

En ese contexto, igualmente entre los asuntos más resaltados y objeto de polémica, fueron el del "vandalismo" contra infraestructuras públicas y privadas ${ }^{8}$ y el de los bloqueos continuados durante cerca de 8 semanas. Estos fueron especialmente fuertes en Cali y el Valle del Cauca, departamento en el que, para mediados del mes de mayo, fecha más crítica, se presentó el $27 \%$, de esta modalidad de protesta, sobre el total del país, convirtiéndose pronto en el referente de la protesta nacional y su dinámica clave para entender el alcance de este momento que marca un quiebre en la expresión de conflictividades, en la protesta social y en las demandas de transformación y cambio social y político.

6 Según el informe producto de la visita de la Comisión Interamericana de Derechos Humanos (CIDH, 2021, p. 6), con fecha de corte al 4 de junio, en Colombia se pudieron calcular desde el 28 de abril, 12.478 protestas en 862 municipios distribuidos en los 32 departamentos. El 89\% (11.060) de estas protestas se llevaron a cabo por medio del uso de repertorios de acción no-violentos tales como: concentraciones en espacios públicos (57,2\%); marchas (20,8\%); bloqueos sin uso de violencia física $(28,8 \%)$ y el $11 \%$ con repertorios violentos.

7 La CIDH visitó al país en el mes de junio y produjo un Informe con diversas observaciones y recomendaciones y entre las principales preocupaciones señaló: el uso desproporcionado de la fuerza; la violencia basada en género en el marco de la protesta; la violencia étnico-racial en el marco de la protesta; la violencia contra periodistas y contra misiones medicas; irregularidades en los traslados por protección; y denuncias de desaparición; así como el uso de la asistencia militar, de las facultades disciplinarias y de la jurisdicción penal militar (CIDH, 2021, p. 7).

8 En Cali con el ataque sistemático al transporte público masivo, fueron quemadas totalmente 13 de las 55 estaciones, quemados 13 buses y el $90 \%$ de las estaciones afectadas, llevando a la parálisis del sistema de transporte masivo MIO, por un espacio cercano a los seis meses, además de la destrucción de parte de la semaforización. Se agrega la destrucción de estaciones de gasolina (64 de las 120 fueron afectadas), bancos y algunos espacios públicos: incendio de alcaldía de Jamundí, del Palacio de Justicia de Tulúa y del peaje de Palmira (Cruz-Hoyos, 2021). A Nivel Nacional, se reportaron saqueos y daños a bienes públicos (2.162) y privados (1.660), daños a infraestructuras policiales (718) y otros hechos violentos (Consejería Presidencial para los Derechos Humanos y Asuntos Internacionales, 2021) 
Como parte de "los bloqueos" en diversos sitios de la ciudad y del Área Metropolitana de Cali ${ }^{9}$ denominados "puntos de resistencia", se produjo la expresión de un heterogéneo "movimiento" juvenil y popular: la Unión de Resistencia Cali (URC)10, constituida sobre la marcha de los acontecimientos, con resonancia y reedición en otras ciudades del país, que llegó a plantear por fuera del Comité local y regional del Paro sus propias reivindicaciones, generando una dinámica que sobrepasó de hecho a la dirección nacional del Paro y en parte a la propia dirección regional.

\section{El conflicto, su dinámica y actores ${ }^{11}$}

En términos de análisis y comprensión del conflicto, debe recordarse que todo conflicto implica actores concretos, enfrentados en torno a un asunto o problema, como objeto de la disputa, que se presenta como una oportunidad de cambio y transformación, pero igualmente en tanto disputa puede escalar hacia fases de enfrentamiento violento, en la cual las partes buscan imponerse o simplemente, debilitar al contrario y/o afianzarse y fortalecerse en sus propósitos. Por consiguiente, las formas de protesta presentes y desarrolladas durante estas semanas, sólo pueden entenderse en tanto formas de acción social, de expresión de "actores" o de grupos con determinadas motivaciones e intereses, en algunos casos directamente conectadas con demandas explícitas -"pliegos petitorios"- pero también no explícitas de naturaleza fundamentalmente políticoideológicas.

En el caso concreto de Cali se ha hecho referencia a los jóvenes y a los estudiantes como actores centrales de la movilización, especialmente en los sitios de bloqueo que terminaron configurando la denominada resistencia y le dieron el carácter de explosión social, determinando en buena parte el impacto del paro en la ciudad, la región y el país y que posicionaron la idea de Cali como capital de la Resistencia (Hernández-Lara, 2021).

Reconociendo su mayoritaria presencia a través de las denominadas "primeras líneas", debe señalarse que son diversas las evidencias de que, igualmente en esos lugares se expresaron y los sostuvieron, desde su inicio, diversos liderazgos, procesos y organizaciones sociales de los territorios y de las comunidades (de iglesias, ONG's, movimientos políticos) que apoyaron la movilización, al menos durante el primer mes. Varios de estos procesos y grupos fueron tomando distancia, en la medida que se sostuvo la estrategia de bloqueo indefinido y que comenzó a afectar a las mismas comunidades: en su movilidad hacia el trabajo, la provisión de bienes, la seguridad en los sectores; esos mismos colectivos, en unos casos discutieron en asambleas de punto, planteando primero la necesidad de flexibilizar - "creación de corredores humanitarios"- y luego el levantamiento de los bloqueos y nuevas estrategias como "barrio adentro" -como en Siloé y Meléndez. En otros casos se fueron alejando y retirando el apoyo, lo que llevaría en parte a un final lánguido, en medio de una extensa discusión sobre la legitimidad de los bloqueos indefinidos ${ }^{12}$ y el carácter indefinido del mismo paro.

$9 \quad$ Incluye municipios aledaños que conectan al Valle del Cauca con el Sur y el Centro del país y representan la tercera área de concentración económica del país: Cali, Jamundí, Palmira, Yumbo, Candelaria, entre otros, con amplios nexos con el norte del Cauca; la mayor parte de sitios de bloqueos se verificaron en el Valle (85 incluyendo 26 en Cali), en puntos neurálgicos para la movilidad en todos estos municipios.

10 Su surgimiento, formación y evolución, son objeto de un texto aparte, resultó de un proceso asambleario, de delegados de las denominadas "primeras líneas", de 28 puntos de resistencia establecidos inicialmente, en particular en una reunión realizada en uno de los auditorios de la sede de Meléndez de la Universidad del Valle, entre los días 8 y 10 de Mayo. No obstante, la producción de su propia plataforma resulta de un proceso de discusión y negociación" que se prolongó varias semanas. El lenguaje, las tácticas y dinámicas de "primeras", "segundas" y "terceras líneas", retoman la "experiencia" de las formaciones de "choque" del estallido en Chile del 2019.

11 Una noción precisa de conflicto implica que este está constituido por actores enfrentados en torno a un problema o asunto objeto de disputa (Entelman, 2005, p. 43), entonces la pregunta es cuáles son los actores y cuál es el asunto objeto de disputa o confrontación. En el caso del Paro Nacional, los dos actores centrales serían el Comité de Paro de un lado y de otro, el Gobierno Nacional y el objeto explícito de disputa, el amplio pliego petitorio; pero en el caso del estallido en Cali se estaría en presencia de un conflicto complejo, multiactor y no un problema sino un conjunto de problemáticas: además de las banderas del paro nacional los puntos diversos presentados especialmente por la URC en el proceso de la misma protesta.

12 Se ha abierto un gran debate sobre si los bloqueos permanentes, representan una forma de protesta pacífica o de acción violenta, denominados por la CIDH denomina "cortes de ruta" (CIDH, 2021, pp. 31-32); el tema debe ser evaluado en el contexto de la confrontación en curso, sus impactos -a quiénes y cómo los afecta- sus actores y la articulación con otras estrategias y las lógicas de acción (no violentas o al contrario, radicales) 
Por otra parte, también se involucraron organizaciones y grupos cercanos a pandillas, "oficinas" y otros actores radicales (milicianos del ELN y de las disidencias de las FARC) que buscaron imponer sus lógicas y su control, lo que fue permanente fuente de tensión y también de cuestionamiento sobre su influencia o incidencia en la movilización, además de propiciar disputas y diversos hechos de violencia adentro y hacia afuera de los puntos $^{13}$.

Esto se relaciona además con el carácter "espontáneo" u "organizado" de la protesta y del estallido, expresada en los "puntos de resistencia": la primera alternativa sostenida por quienes la ven como una respuesta "heroica" y casi de supervivencia de los jóvenes contra la "desigualdad estructural". La segunda, del lado gubernamental y de críticos especialmente de los "medios violentos del paro", para quienes detrás había intereses de grupos organizados, incluyendo movimientos políticos, algunos cercanos a expresiones ilegales. Las evidencias se sitúan a mitad de camino, en efecto, varios activistas del paro y de los puntos de bloqueos, reconocen la existencia de un proceso de preparación previo, que de alguna forma remiten al paro del 21 de Noviembre de 2019, cuando se presentaron bloqueos en algunos de los puntos que reaparecieron en 202114, pero también a hechos previos, sucedidos en la Universidad del Valle, en Abril de 201915.

En este sentido, no puede descartarse como hipótesis que, si bien el estallido expresó la irrupción de dinámicas estructurales ${ }^{16}$ que hicieron eclosión en la coyuntura creada por la pandemia del Covid y su manejo por parte del Estado, la convocatoria del Paro abrió el espacio para que se expresaran, de un lado, actores espontáneos -especialmente muchos de los jóvenes y grupos marginados- y de otro, para que actuaran y confluyeran procesos organizados, agendas e intereses no espontáneos, que aprovecharon la coyuntura y convirtieron el paro y su dinámica en un espacio de acción política, con propósitos diversos y complejos, particularmente en el caso de Cali y el Valle.

Como parte de esta hipótesis la escalada de la violencia especialmente fuerte en Cali - como en Bogotápodría también leerse como una especie de "teatro de operaciones", en el que actores que incentivaron y apoyaron las acciones de hecho y de violencia en la ciudad, la convirtieron en un "laboratorio", un campo no sólo de "aprendizaje", sino en el que se jugaban intereses políticos, asociados a la explotación de los efectos de la escalada violenta: confrontar a la autoridad y su legitimidad, "agudizar las contradicciones" o también generar la sensación de inseguridad, caos, incertidumbre y miedo, que tiene como consecuencia demandar más "autoridad y orden"; estrategias y repertorios en las que convergen y se encuentran sectores extremistas de izquierda y de derecha ${ }^{17}$.

13 El tema fue especialmente crítico en el sitio más emblemático: Puerto Resistencia y el último en ser levantado en una mezcla de presión policial y negociación, a finales del mes de Julio. También en otros puntos se presentaron situaciones similares: Paso del Aguante, Puerto Madera y Meléndez.

14 En los sucesos de Noviembre de 2019, cuando se inició el desbordamiento de la protesta y se comenzaron los bloqueos, el Alcalde Mauricio Armitage (2016-2019) decretó el toque de queda y se logró rápidamente levantar los bloqueos; también se dieron diversos hechos de vandalismo e intentos de saqueo y asalto a unidades residenciales de sectores medios y populares, algo parecido sucedió un día después en Bogotá. A pesar de que el Alcalde de Cali y su secretario de Seguridad inquirieron en el Puesto de Mando Unificado PMU de la policía, sobre lo qué estaba pasando, y quienes estaban promoviendo estos hechos, y "qué explicación tenían las fuerzas de seguridad, porque todo era muy raro", nunca hubo respuestas. Entrevista con exsubsecretario de Seguridad del Gobierno Armitage.

15 En abril de 2019, se concentraron diversos grupos de la ciudad -milicias, barras bravas, pandillas- y también algunos grupos radicales del movimiento estudiantil, para desarrollar una jornada de protesta desde el campus de Meléndez de Univalle, contra el Gobierno Duque, en un número superior a 200 activistas, encapuchados en su mayoría, confrontaron al ESMAD en las afueras de Ciudad Universitaria en un largo combate con extenso uso de "papas-bombas". Como resultado y en una acción accidental, estalla un maletín con esos artefactos, produciendo la muerte de un estudiante de otra universidad de la ciudad y dos más resultaron con fuertes heridas y mutilaciones. Se trató de una acción mancomunada de grupos que utilizaron repertorios violentos y realizaron un verdadero ensayo de combate urbano.

16 Pero estas no se refieren exclusivamente a la "estructura económica", sino a aspectos más sistémicos: de gobernabilidad, el rezago educativo, la conflictividad territorial y las problemáticas de seguridad del suroccidente del país, recicladas en Pistas para entender el 'estallido social' en Cali (Álvarez-Rodríguez, 2021).

17 Este asunto no es ajeno a la disputa y la polarización política que se viene expresando, desde 2018, con mucha fuerza en el país y especialmente en Cali y el suroccidente, que domina este momento y dominará el ambiente de las elecciones presidenciales del primer semestre de 2022, con una alta probabilidad de que se termine el largo período de hegemonía del Centro Democrático y del Uribismo y la factible victoria de la oposición en cabeza de Gustavo Petro. A través de las redes circularon acusaciones que asociaban la protesta y los bloqueos a una estrategia "petrista", mientras el líder de la Colombia Humana le dijo al Comité de Paro que debieron haber frenado las manifestaciones (Semana.com, 2021). 
Retomando el tema sobre la "naturaleza" de la movilización, también se presentaron hechos espontáneos relacionados con la respuesta ciudadana y de personas en las comunidades, que le dieron impulso y legitimidad a la protesta con dinámicas que desbordaban a sus líderes, incluyendo la circulación de grupos y la alternancia en los "liderazgos", en los diversos sitios y conforme a los momentos, que ratificaban parte de esa espontaneidad, que al mismo tiempo representaría parte de su debilidad.

La pluralidad y diversidad de actores, con agendas e intereses diversos, señalan la complejidad de motivaciones y factores que le dieron permanencia a la "explosión" en la ciudad pero también explican las dificultades posteriores para canalizar el proceso y encontrar alternativas y salidas. Esas dificultades se expresan en el proceso mismo de conformación y acción de la Unión de Resistencia Cali (URC), la designación de sus vocerías y la determinación de una plataforma de exigencias, que se vino a consolidar sobre la marcha en la propia fase de negociación con el Gobierno Distrital ${ }^{18}$. El carácter heterogéneo, en términos de expectativas, experiencias y posturas ideológico-políticas de este colectivo, así como las tensiones políticas y la dificultad de consolidar vocería y liderazgo estable y reconocido, incidieron en el diálogo y la negociación entre ellos y con otros sectores -gubernamentales, empresariales, universidades, eclesiales etc. Asunto clave a la hora de concretar sus logros en la negociación y conducir a un cierre concertado y no a la imagen del levantamiento de los bloqueos por cansancio o agotamiento ${ }^{19}$.

Este mismo tema puede indicar la expresión de un conflicto "difuso" y por momento "intratable" (por la diversidad de problemáticas, la dispersión de la contraparte y de los puntos objeto de la disputa); asunto que parece fundamental para encontrar no sólo respuestas y alternativas de coyuntura, sino sobre todo de transformación, superando las lógicas que tendían a mantenerlo en la confrontación violenta y su instrumentalización política ${ }^{20}$.

Esta cuestión se vincula al impacto económico del Paro, que alimentó buena parte del debate y la polarización respecto de la legitimidad de los bloqueos -fuertes en Cali y el Valle del Cauca- como forma de protesta pacífica, en tanto estos si bien se constituyeron en una poderosa arma de presión contra el gobierno y en parte contra los sectores empresariales, terminaron afectando a las propias comunidades y a personas de los sectores más pobres, que precisamente habiendo apoyado al movimiento fueron tomando distancia y discutiendo la necesidad de su levantamiento.

Los impactos del Paro fueron diversos ${ }^{21}$, algunos muy cercanos a la vivencia de la gente como el desabastecimiento, nunca antes vivido en la ciudad, de bienes básicos (gasolina, alimentos y materias primas

18 Los pliegos y demandas fueron diversos, el primero surgió a inicios de mayo de 2021 e incluyó: 1. Garantías y exigencias (cese de la asistencia militar, renuncia del Ministro de Defensa y los comandantes generales del Ejército y la Policía, aceptación de responsabilidad del gobierno nacional, liberación de detenidos, visita de organismos internacionales y desmonte del ESMAD), 2. Reformas constitucionales (tributaria, gasto público, edad para el voto, laboral, no realización de fumigaciones y del fracking, reforma de la policía, ley de cuotas entre otras), 3. De nivel local, comisión de paz, de verdad y esclarecimiento, programas de educación, cultura, empleo y emprendimiento (Documento entregado por delegados de 18 puntos el 10 de mayo de 2021). No obstante, en el mes de Septiembre en la fase avanzada de la Mesa de Negociación la URC entregó una matriz de propuestas centradas en lo local y en seis temas (Organización social, Garantía de derechos humanos, Esclarecimiento de la verdad, Empleo y productividad, Seguridad alimentaria, Salud y educación y cultura), con un total de 24 puntos y un cronograma que inicia desde finales del 2021 y va hasta el año 2023.

19 El levantamiento y la flexibilización de los bloqueos fue planteado inicialmente en la Mesa de Negociación por uno de los delegados del Alcalde, recibiendo el rechazo inicial de delegados y asesores de la URC, de esta forma el proceso de levantamiento tuvo una dinámica determinada por discusiones y tensiones en la Mesa y los diferentes sitios, iniciado con discusiones o reuniones asamblearias, contactos con representantes del gobierno local y la mediación de delegados de la iglesia, de organismos internacionales (ONU, MAPP-OEA) y de organizaciones de derechos humanos, diálogos con los grupos más activos y líderes sociales con incidencia en los sitios. También estuvo siempre presente una estrategia disuasiva y de presión de la Policía, pero finalmente sólo en cuatro o cinco puntos esta se convirtió en la estrategia determinante. Estrategia precedida por la amenaza del comandante general Zapateiro en el sentido de que en pocos días lograría el despeje de los bloqueos.

20 Este asunto plantea un debate relevante no sólo respecto de este momento, sino de la protesta social y en particular de la protesta pacífica que, como señala (Sánchez-Salcedo, 2021), remite a un asunto de cultura política, en tanto se verifica la propensión a la utilización de repertorios violentos tanto del lado estatal, como de "los jóvenes"; pero igualmente la instrumentalización por sectores, que en unos casos aprovechan la protesta social, para desplegar repertorios de violencia contra el Estado y el "orden establecido", con preparación previa y una logística propia de sectores más radicales (milicias, ELN, disidencias) y de otro grupos de derecha, interesados en mantener el caos, la tensión y el reclamo de más "autoridad y orden".

21 En los primeros 15 días del paro, el costo estimado por el Gobierno Nacional era de \$484 mil millones de pesos diarios (US\$128,000/ día) y Fedesarrollo estimó que en el mes de mayo el impacto habría estado entre \$4,8 y \$6 billones (US\$1286 millones y US\$ 1600 millones), y que para el Valle del Cauca las pérdidas equivalían a la tercera parte del total (El Espectador, 2021). La 
diversas), que se tradujo en: el incremento de precios, la fractura de algunas cadenas productivas, la imposibilidad de movilizarse al trabajo -formal o informal - el consecuente deterioro de fuentes de ingreso de muchas familias, y el crecimiento de la problemática del hambre que afectaba ya antes especialmente a los sectores más pobres de la ciudad.

\section{¿Las comunidades y la sociedad civil, un tercero en la disputa ${ }^{22}$ ?}

La coyuntura y el desenlace del Paro, produjo un gran sacudón y un profundo impacto político y emocional con la movilización de conciencia de amplios sectores sociales, que involucró de diversa manera a toda la sociedad caleña y regional, agitando en las primeras semanas sobre todo las pasiones y las emociones, incluyendo el miedo y una sensación de desesperanza y fractura irremediable. En efecto, en las primeras semanas, diversos sectores ciudadanos reaccionaron primero contra los bloqueos y contra "el vandalismo", la mayoría en forma pacífica, llegando muchos a los puntos a dialogar 23 , con acciones no violentas, que muy poco se mostraron en la información y en las narrativas predominantes en las redes.

Pero por otra parte en la zona Sur de la ciudad, algunas personas esgrimieron y utilizaron armas contra manifestantes, en el enfrentamiento entre grupos de la Minga Indígena ${ }^{24}$ y personas de Ciudad Jardín, el día 9 de mayo, cerca de la Iglesia la María en Ciudad Jardín, que pudo haber llevado no sólo al escalamiento del enfrentamiento, sino a una especie de confrontación entre sectores civiles en la ciudad; evento precedido además por la amenaza por parte de algunos habitantes de esta zona contra quienes sostenían los bloqueos. Estos hechos dieron el calificativo de "los ciudadanos de bien", para referirse a grupos de ciudadanos de algunos barrios de estratos altos opuestos al paro y quienes convocaron a la "marcha del silencio" contra el paro y los bloqueos, realizada el 25 de mayo de $2021^{25}$.

Como resultado, en la narrativa expresada en diversos medios -prensa y especialmente en las redes sociales- se ha resaltado el tema de la polarización política, ideológica y cultural, con nuevos estigmas ("los ciudadanos de bien", "los vándalos" o "los indios") 26 y nuevas "fronteras invisibles" 27, precisamente entre barrios de estratos altos y sectores populares aledaños. Si bien esta reacción fue evidente y muy fuerte en las primeras semanas y se mantiene como un asunto no sólo de polémica, sino de confrontación ideológica alentada por sectores extremistas, también motivó la expresión de voces y de una dinámica de reconocimiento, de escucha -entre diversos grupos y liderazgos ciudadanos- y procesos orientados no sólo a entender las motivaciones y razones en la base de la "indignación", sino la profundidad de las desigualdades, que dividen a la sociedad, y la necesidad de construir caminos de inclusión, de transformación y reconciliación. Iniciativas de sectores de la sociedad civil que contribuyeron en la fase crítica, precisamente a desescalar la confrontación

Cámara de Comercio de Cali, señaló el 27 de mayo que el 52\% de la capacidad productiva del Valle y el Cauca fue paralizada. ACOPI (Asociación Colombiana de pequeños industriales) informó que en el 77,3\% de sus empresas tuvieron alto impacto: "más Mas allá del costo económico estimado dentro un ciclo de 51 días, en que se vivió con mayor intensidad los efectos del paro nacional y la alteración del orden público, la incertidumbre sobre el clima de negocios, la destrucción de empleo es el resultado de estas movilizaciones violentas" (ACOPI, 2021).

22 Esta noción hace referencia a la intervención o la implicación de un tercero en un conflicto -como aliado, como parte interesada en una u otra dirección, como mediador o como incentivador del enfrentamiento o de la búsqueda de soluciones (Entelman, 2005, p. 133); "tercero" que puede ser relevante en el rumbo que tome la confrontación, o la solución del conflicto.

23 Un caso emblemático fue el de empresarios del Grupo Multisectorial, que desde los primeros días se fueron a los puntos a dialogar con los jóvenes, a escuchar sus razones, a tratar de entenderlos y que en estos meses desarrollaron cerca de 12 conversatorios masivos -virtuales- con la participación de los y las jóvenes de primera línea sobre temas diversos: educación, empleo, salud, empleabilidad etc. (Entrevista y participación con lideres de este grupo Gustavo Muñoz, Kees Estapel, entre otros).

24 La presencia y el papel de la Minga, como otro actor, merece un análisis detenido pues más allá de la misión enunciada por sus líderes de solidaridad con protección y seguridad con los manifestantes, no puede entenderse por fuera de su agenda política territorial y respecto del Gobierno y el Estado nacional.

25 Fue evidente la incidencia de parlamentarios del Centro Democrático en esta convocatoria e igualmente de quienes promovían la revocatoria del Alcalde Jorge Iván Ospina, que revelaba también los intereses políticos que se movían en este contexto y en particular la oposición al Gobierno local.

26 Uno de los hechos más emblemáticos que disparó pasiones y un gran debate ideológico y cultural, fue el derribamiento de la estatua de Sebastián de Belalcazar -fundador de la ciudad en el año 1536- por miembros de la comunidad indígena Misak, el día 28 de abril, considerada por algunos sectores de la ciudad como un estatua emblemática de la "caleñidad" y su abatimiento como una "afrenta a la ciudad", pero que en la perspectiva de los indígenas hace parte de su lucha por la "descolonización", y la denuncia del genocidio de las comunidades originarias, cometido por los conquistadores españoles.

27 Pero igualmente en medio de la protesta cayeron y se diluyeron algunas de las fronteras invisibles que llevaban a enfrentamientos entre los jóvenes de pandillas, de barrios o sectores en sectores populares, o entre las "barras bravas" de los dos equipos de fútbol de la ciudad: el América y el Cali. 
y a lograr que las estrategias de diálogo y negociación, contribuyeran a canalizar la protesta hacia una salida no violenta y en perspectiva de transformación pacífica de la conflictividad, tanto en escenarios institucionales (Mesa de negociación entre representantes gubernamentales y la URC), como en otros escenarios ciudadanos con la participación de jóvenes, empresarios, líderes sociales, académicos y de la iglesia.

En esta dirección hago referencia sintética a algunas dinámicas y actores que considero, caracterizan esta fase de transición, luego de ocho meses de iniciado el paro y de seis meses de su fase de mayor escalamiento, buscando resaltar precisamente una dimensión menos presente en la narrativa dominante del paro -la del "heroísmo", la "resistencia" y el "vandalismo" - en perspectiva de giro epistemológico de los estudios de paz, que precisamente muestran el activismo y la "agencia" hacia la salida no violenta y la construcción de salidas pacíficas y transformadoras al conflicto y conflictividades ${ }^{28}$.

Algunos procesos emblemáticos se fueron consolidando en estos meses y hacen parte del contexto de dinámicas que favorecieron los procesos de diálogo y de acciones transformadoras que rodearon el espacio político-institucional representado en la Mesa de Negociación entre el Gobierno Distrital de Cali y la Unión de Resistencia Cali, a la que me refiero en la parte final. Entre esas están las siguientes: Mediación por Cali, Compromiso Valle del Cauca ${ }^{29}$, El Acuerdo por Cali y su propuesta de construcción de una Visión compartida de ciudad, como proyecto estratégico de transformación de la conflictividad, de inclusión y reconciliación ${ }^{30}$, la movilización desde el sector Universitario (Mesas de trabajo de Cidesco, y la Universidad Pal Barrio de estudiantes y profesores de Univalle) y otras iniciativas más localizadas, pero igualmente importantes, en cuanto al activismo social, en tanto procesos de empoderamiento pacifistas (Muñoz-Muñoz, Herrera-Flores, MolinaRueda y Sánchez-Fernández, 2005) ${ }^{31}$.

En primer lugar debe señalarse que esta movilización impuso el llamado a la defensa de la vida por encima de todo ${ }^{32}$ y la no violencia, que realmente logró que el escalamiento y el nivel de la confrontación y violencia no hubieran sido más devastadores; abriéndole paso a una idea fuerza: "lo valiente es dialogar" levantada precisamente por los rectores de las Universidades de la región agrupadas en Cidesco, y el Arzobispo de Cali, propuesta directamente en el espacio de "mediación por Cali".

La iniciativa de Mediación por Cali33, surge como espacio no-formal, en los primeros días del mes de mayo, a instancias de un primer colectivo de trabajo conformada por algunos académicos y líderes empresariales, propuesta al Arzobispo de Cali, quien la lidera y en la cual se convoca a lideres empresariales regionales, religiosos, ambientalistas, académicos, mujeres y jóvenes de la URC, asesores de la Alcaldía de Cali y la Gobernación del Valle, con una agenda asociada a entender las razones del paro, a escuchar a sus diversos actores, y apoyar el diálogo entre los y las jóvenes, el alcalde y sus secretarios, empresarios, líderes sociales, representantes de la Minga. También a buscar alternativas y proponer acciones transformadoras en diversos planos.

28 Las iniciativas y procesos se reseñan con base en la observación participante, del autor y del equipo del Instituto, presente durante el Paro, en varios de los espacios, en diálogos con actores de los mismos, la Mesa de Negociación entre la Alcaldía y la URC, la Mesa de Cidesco, conformada a instancias de la coyuntura, y del Instituto de Estudios Interculturales de la Pontificia Universidad Javeriana de Cali, entre otros.

29 Impulsada y liderada por el grupo de empresas más fuertes económicamente de la región y sus organizaciones gremiales, para constituir un fondo para programas sociales en temas prioritarios resaltados en la protesta en Cali. https://www.compromisovalle.org.

30 Iniciativa planteada por personas de diversa procedencia, con trayectorias y realizaciones a nivel local y regional: empresarios, trabajadores de la cultura, comunicadores, académicos, jóvenes, columnistas, exfuncionarios públicos, construida en un proceso de diálogo desde el mes de mayo de 2021 y lanzada el 8 de Agosto de 2021, y firmada inicialmente por 308 personas de un amplio espectro social, político y cultural.

31 Este punto merece un trabajo aparte en tanto, se han multiplicado las expresiones precisamente en la perspectiva de acciones constructoras de diversas paces, algunas de grupos de ciudadanos - de los mismos sectores, señalados irónicamente como ciudadanos de bien- como las Iniciativas de paz del oeste de Cali (El país, 2021) para movilizar el diálogo y el reconocimiento entre personas de los estratos altos del área oeste de la ciudad y jóvenes de la Comuna 1 ( La Portada); otras para promover la reflexión y aprendizajes entre las organizaciones sociales y grupos de las comunidades (Cali Distrito que Renace).

32 Idea levantada desde las primeras reuniones por el Arzobispo de Cali, en el espacio de Mediación por Cali.

33 La primera reunión se realizó el 5 de Mayo a las 7 am en formato virtual, con una asistencia inicial de unas veinte personas, pero que fue creciendo en las semanas más críticas, con la asistencia promedio de 70 y llegó hasta 120 personas, con reuniones semanales cada martes a las 6:00 a.m. en modalidad virtual. 
Algunos logros de este proceso: la propuesta de los corredores humanitarios, para que en diversos puntos de bloqueos se permitiera, primero el tránsito de heridos, enfermos y posteriormente de productos esenciales; escuchar directamente a algunos representantes de jóvenes de las primeras líneas, para entender sus razones, demandas y expectativas y posibilitar la propia labor de mediación; escuchar y dialogar con el Alcalde y miembros de su equipo de gobierno, sobre su propio análisis, propuestas y alternativas. Estos diálogos abrieron espacios para afinar respuestas frente a temas o asuntos más sentidos y críticos planteados por los representantes de la URC -educación, empleabilidad, comedores comunitarios y especialmente la protección y atención en derechos humanos- contribuyendo a algunas propuestas que la Alcaldía tradujo en medidas permanentes como el Programa Todos y Todas a Estudiar 34; también la mediación exitosa, entre la Minga y ciudadanos de Ciudad Jardín, ante los temores levantados por su llegada en el 19 de diciembre a Cali.

Debe señalarse que, en este espacio no todo era diálogo fluido y "color de rosa", sino al contrario, allí se expresaba la desconfianza hacia y entre los diversos actores, la polarización, y por momentos la pugnacidad y la intolerancia, a veces en forma abierta y otras en formas más sutiles y veladas. No obstante, finalmente las personas participantes mantuvieron la disposición y la permanencia, que permitiría en estos meses ganar el respeto mutuo y el reconocimiento, y un nivel de confianza básica y de "acuerdos", que marcan en el proceso una ruta, que uno de sus participantes denominó la de la "Cali resiliente".

En síntesis, la iniciativa "Mediación por Cali" se dio en medio de diversas actividades de diálogo social

y político ${ }^{35}$, en las cuales se expresó el renacimiento del activismo desde sectores ciudadanos y de la sociedad civil local ${ }^{36}$, sea como fuerza de presión e interpelación especialmente a la institucionalidad pública -Gobiernos local, regional y nacional-pero por momentos con acciones de interlocución con los propios jóvenes ${ }^{37}$.

\section{El Diálogo político, como estrategia y espacio de transformación del conflicto}

Las acciones reseñadas antes se pueden enunciar, desde una perspectiva teórica, que va entre la resolución y la transformación del conflicto 38 , que implica en primer lugar asumir y entender el conflicto como una oportunidad de cambio y transformación y, en segundo lugar de movilización y articulación de iniciativas (institucionales, ciudadanas, comunitarias), con la participación de actores directos y terceros. El diálogo y la interlocución política aparecen como estrategias de reconocimiento y comprensión de los puntos de vista enfrentados y espacios de comunicación para construir confianzas, analizar alternativas y construir acuerdos, no sólo para desescalar la confrontación sino para satisfacer demandas de corto plazo y modificar las condiciones que nutren el conflicto y la protesta misma.

En este contexto, el escenario central, y que tendría un papel decisivo en la canalización del estallido en Cali, fue la apertura por parte de la Alcaldía Distrital, de un espacio de diálogo, y al mismo tiempo de reconocimiento, con la recién constituida URC, como interlocutor y contraparte de la protesta en la ciudad de Cali; ese espacio fue construido con la mediación de representantes de Naciones Unidas y la Arquidiócesis de

34 Lanzado por la Alcaldía el día 29 de Noviembre y sustentado en el Acuerdo 0509 de 2021.

35 En estos meses hubo reuniones y diálogos entre actores diversos, tanto con el Gobierno nacional y sus representantes, como con el gobierno Departamental y el propio Gobierno local, igualmente una presencia muy activa de delegaciones y representantes de la Comunidad Internacional delegaciones de la ONU, Mapp-OEA, Embajadores de la UE y de USA, y también con el respaldo y activa participación de la Arquidiócesis de Cali, entre otros.

36 Partimos de una noción de sociedad civil, en tanto grupos de ciudadanos organizados y que se expresan en torno a asuntos de interés público y común y no simplemente como individuos; en concreto puede reseñarse el protagonismo del Grupo Intersectorial (sector empresarial), del Comité Intergremial del Valle, desde donde se viabilizó la iniciativa Compromiso Valle; las diversas iglesias y en particular la Arquidiócesis de Cali y su programa de Pastoral Social, la Junta de rectores de CIDESCO -red que agrupa a las universidades públicas y privadas de la región- ONG's locales con trabajo e incidencia en procesos comunitarios y otras.

37 No obstante la afirmación expresada por representantes de algunos grupos empresariales, en medio de la fase dura de los bloqueos, en el sentido de que no se reunirían con "encapuchados" y delegados de la URC, en la cuarta semana de mayo, se realizó una reunión ( un "diálogo improbable"), con la mediación de la Arquidiócesis, entre delegados de la URC y representantes del sector empresarial y de la iniciativa Compromiso Valle, un diálogo franco, sobre las razones y posturas de la URC pero igualmente sobre la visión y la propuesta del sector empresarial.

38 La diferenciación y la relación entre estos dos procesos es formulada con Jean P. Lederach, en tanto la primera se plantea esencialmente para desescalar el conflicto y en especial la violencia y apunta a acuerdos de corto plazo, en tanto la segunda, que tiene una perspectiva de largo plazo, busca responder a factores más estructurales y a producir cambio en las relaciones entre los actores (Maiese y Lederach, 2004) 
Cali, en las que además estuvieron presentes el Ministerio Público y delegados de la Minga y organizaciones sociales locales ${ }^{39}$.

Como resultado de este proceso y teniendo entre sus antecedentes el Decreto 1.17.0517 de 2021 de la Gobernación del Valle del Cauca, que dispuso la creación de "mesas territoriales para el desarrollo social en el contexto del Paro Nacional del 28 de abril de 2021" 40, el Alcalde Distrital expidió el Decreto 4112.010.20.0304 de 2021, en el cual reconocía en primer lugar a la URC como "movimiento autónomo de articulación de los puntos de resistencia para la interlocución en el diálogo social con la institucionalidad y la sociedad" (Artículo $1^{\circ}$ ) y además establecía: Garantías para la protesta pacífica (Artículo $2^{\circ}$ ), un Canal de salvaguarda de vidas, una Comisión de Derechos Humanos y una Mesa de diálogo, "como un espacio inclusivo y participativo, para la definición de agendas que nos conduzcan de manera concertada a distensionar las dificultades, trazar líneas de inclusión social y brindar garantías de participación".

El Decreto recibió ataques de diversos sectores y especialmente de representantes del Centro Democrático -el partido del gobierno- y grupos locales opuestos y enfrentados con el Gobierno Distrital, por considerar que el reconocimiento y la negociación con la URC, legitimaba la violencia y el vandalismo en la ciudad. Simultáneamente fue objeto de cuestionamiento por el Ministerio Público, y demandado ante el Juzgado Administrativo de Cali, en tanto el decreto estaría "amparando las acciones de hecho e impidiendo la acción legítima de la policía y adicionalmente, contraviniendo el Decreto presidencial 575 de 2021"-sobre orden público y "asistencia militar a alcaldes y gobernadores" - señalándolo

como atentado grave e inminente contra la institucionalidad constitucional y legal, lo que generaría no sólo una desfragmentación de la unidad nacional, sino también una afectación grave y directa a los derechos de terceros que se verían afectados por el impedimento de la fuerza pública para actuar frente alteraciones del orden público. (Auto 603016-2021)

Como resultado de la medida cautelar se suspendió el Decreto; no obstante una tutela interpuesta restablecería su aplicación.

Así, cobró vida el camino de diálogo y negociación política-institucional41, casi un mes después de iniciada la protesta, en un contexto y como un proceso intrincado y complejo. En primer lugar porque, la URC planteó -no sin razón- como asunto prioritario y por momentos una precondición para avanzar en las negociaciones de otros temas, la denuncia de violaciones de derechos humanos y la demanda de verdad y justicia; lo que en parte desbordaba la competencia del Gobierno Distrital. La continuidad de los enfrentamientos y de hechos de violencia en varios de los "puntos de resistencia", así como la denuncia de detenciones y seguimientos a miembros de la URC, implicaron un motivo de permanente tensión y freno a una negociación productiva, en los primeros dos meses de su trabajo.

De otra parte, dificultades de los dos actores centrales, operaron como obstáculos para el avance de una negociación productiva; de un lado, la propia naturaleza de la URC, como grupo heterogéneo, con vocerías inestables, circulantes y oscilantes en sus propias posturas, a veces dominadas por la posición de asesores, con sus propias agendas políticas, que seguían alimentando el radicalismo no sólo verbal, sino que de hecho justificaba y mantenía los bloqueos cuando era evidente el desgaste y el alto riesgo para quienes los realizaban y el riesgo político para la propia mesa y el camino de la negociación. Se agregan las propias dificultades del lado del Gobierno local y en parte del Departamental, en consolidar una propuesta y una ruta que pudiera responder eficazmente tanto a la presión por levantar los bloqueos, recuperar el control del orden público en la ciudad y responder a las exigencias inmediatas de los voceros y colectivos: seguridad y justicia, protección,

39 El autor de este artículo participó como uno de los dos observadores -en representación de CIDESCO, sector académico- en la Mesa.

40 El Decreto servía de marco para mesas similares en los 40 municipios y dos distritos especiales del departamento del Valle del Cauca se proponía: "la construcción colectiva de una ruta de concertación y garantías, concebido además como instancia de participación comunitaria que se articulará al GRAN PACTO PARA LA JUVENTUD DE COLOMBIA liderado por el Gobierno Nacional"; además, en su artículo segundo estableció como objetivo: "la identificación y análisis de las problemáticas sociales que permitan concertar la ruta de los posibles escenarios de solución y acompañará todas las acciones reivindicatorias provenientes tanto de los Gobiernos Nacional, Departamental, Distrital y Municipal, así como demás actores públicos y privados que aporte en la solución de las demandas" (Decreto 1.17.0517 de 2021)

41 Estrategia resaltada y valorada por la ONU, desarrollada no sólo en Cali sino en Bogotá, Medellín y varios Departamentos (Caquetá, Huila, Nariño, entre otros). Oficina del Alto Comisionado de las Naciones Unidas (ACNUDH, 2021). 
Álvarez-Rodríguez

alimentación, empleabilidad y alternativas de educación, entre otras. En un contexto político no favorable: tensiones con el gobierno central -más interesado en una salida de fuerza-, el reclamo de diversos sectores ciudadanos afectados por los impactos del Paro, y que desde diferentes posturas confrontaban al Alcalde Distrital ${ }^{42}$, y el condicional apoyo político en el Concejo Distrital ${ }^{43}$ para sacar adelante las propuestas para dar respuestas al menos a una parte de las demandas planteadas en la mesa de negociación.

Una mezcla de desgaste, cansancio de los actores centrales, también de necesidad y simultáneamente el cambio de perspectivas, para encontrar una ruta de avance permitió que se fueran concretando algunos logros, de carácter inmediato y otros más mediatos, unos desarrollados directamente por el gobierno Distrital, que finalmente logró el apoyo y la aprobación del Concejo y levantar como eje de su propuesta la "reconstrucción de la casa común" en el contexto del proceso de la adecuación de la nueva estructura del Distrito Especial, Deportivo, Cultural, Turístico, Empresarial y de servicios y la definición de nuevas localidades ${ }^{44}$. De esta forma, el espacio del diálogo y la política logró un "cierre parcial", con una perspectiva de resolución temporal y en parte de transformación pacífica del conflicto, que incluyó algunas conquistas y logros parciales para los y las jóvenes ${ }^{45}$ y las comunidades y un terreno de aprendizaje y dinamización política de la ciudad.

\section{Una hipótesis a manera de conclusión}

Transcurridos ocho meses, podemos arriesgar una hipótesis en relación con la situación de la ciudad, sacudida por el estallido: se ha producido un salto de conciencia expresado en diferentes sectores y acciones, que puede traducirse en una perspectiva de transformación pacífica, a partir del diálogo múltiple, el cumplimiento de los acuerdos parciales, el desarrollo y profundización de acciones transformadoras diversas desde el sector público, los empresarios, las universidades, la comunidad internacional y sectores de los jóvenes de la URC y las organizaciones sociales. Que le han dado paso a la idea fuerza de "Cali resiliente", y muestran también un proceso de politización colectiva que privilegia, precisamente la acción política, la no violencia y el diálogo entre los distintos actores. Se trata de un proceso abierto y en el que se expresan liderazgos ${ }^{46}$ y motivaciones diversas, emergentes y nuevas en el contexto local; proceso contradictorio, con fragilidades y amenazas/retos para consolidar y sobre todo profundizar la perspectiva de convergencia hacia el propósito de construir visión y estrategias de transformación económica, social, política y cultural, para superar desigualdades y exclusiones históricas.

En este sentido, son grandes los retos que se enfrentan, que de no ser asumidos y transformados con acciones cooperativas y constructivas, representan un riesgo de reedición, en una fase de la historia local y nacional, plagada de incertidumbres. En tanto el "estallido" ha revelado los problemas de liderazgo político y gobernabilidad local: las debilidades y la baja capacidad de gestión del lado gubernamental, por la lentitud y limitada eficacia en las políticas públicas y acciones a su cargo; la desconfianza hacia el Gobierno local, de parte de actores claves -empresarios y élites locales y por momentos los mismos jóvenes y sectores académicos-, pero no sólo por el tema de transparencia; a lo que se añade el desconocimiento mutuo. Esto se traduce en la ausencia de una apuesta común, de largo plazo y la baja capacidad de coordinación para responder no sólo con acciones de corto plazo sino con políticas públicas y proyectos de transformación estratégica.

42 Entre esas la promoción de iniciativas de revocatoria y la oposición cerrada por parte del partido Centro Democrático y algunos de sus líderes nacionales y regionales, especialmente con la acusación de haber entregado la ciudad al vandalismo.

43 Un asunto notable en todo este proceso fue el "mutis por el foro", por parte de la clase política local, en términos de buscar respuestas y acompañar una salida, con la excepción del partido Centro Democrático, centrado en hacer oposición y explotar la coyuntura acusando al alcalde de ser cómplice de la protesta y de los vándalos.

44 Giro expresado en reunión extraordinaria de la Mesa de Negociación entre el Alcalde y la URC, el 16 de Septiembre de 2021, en el despacho de la Alcaldía, donde ambos sectores planearon una apertura y la necesidad de concretar puntos del Acuerdo, resolviendo problemas de planeación y adecuada gestión desde la Alcaldía, pero también redimensionando y concretando las expectativas del lado de los jóvenes, en cuanto a puntos y tiempos (mediano plazo). (Resumen de balance de propuestas desde la URC. PDF, 16/09/21)

45 En particular pueden señalarse el Programa Todos y todas a estudiar promulgado por la Alcaldía y que beneficiará a 10 mil jóvenes en los próximos dos años, y diversas iniciativas de empleabilidad desarrolladas desde los Gobiernos Local y Departamental y el sector empresarial y, la revitalización de la estrategia de comedores comunitarios e iniciativa de huertas urbanas en perspectiva de soberanía alimentaria; varios de esos impulsados por Compromiso Valle -www.compromisovalle.org-, dirigidos a las comunidades, a organizaciones sociales y a los jóvenes, con impacto local y regional, importante.

46 En este sentido debe señalarse que en la primera elección nacional de los Consejos Municipales de Juventud el 29/11/21, tres listas de Cali tuvieron un resultado destacado a nivel nacional, entre esas la lista Unidad Popular-Cali en Resistencia vinculados a la URC, si bien con una baja votación absoluta (235 votos), confirmando el interés por una mayor participación y reconocimiento político, expresado en diferentes espacios en el contexto del paro en Cali (Diario Occidente, 2021, p. 3). 
Por otra parte, la conformación y la actuación del propio actor o contraparte social (en primer lugar los jóvenes y la URC, pero también las organizaciones sociales y ciudadanas) requieren superar no sólo fracturas y la polarización, sino los problemas de liderazgo y capacidad de representar la pluralidad de grupos expresados localmente. En este sentido es un reto fundamental consolidar nuevos liderazgos y el protagonismo expresado desde diferentes grupos de jóvenes y ciudadanos, incluyendo la renovación de la política local, el fortalecimiento de las capacidades de diálogo y escucha para la construcción de acuerdos, precisamente en dirección a construir un proyecto y una visión de ciudad incluyente, como guías del compromiso y la acción colectivos.

El asunto de fondo es la permanencia de condiciones estructurales -que como he señalado se refieren no sólo a los aspectos económicos, sino de gobernabilidad, de seguridad, y al profundo rezago educativo, que propiciaron la protesta y el estallido- pero sobre todo la percepción de inacción o desconfianza en la puesta en marcha de acciones y procesos efectivos de cambio. En este sentido, un reto central se refiere a la permanencia de las amenazas y riesgos de seguridad, que se expresan en la ciudad y la región, vinculada a la presencia de actores ilegales - bandas del narcotráfico, la criminalidad y la insurgencia-47, actuantes en el estallido y que tienen a la ciudad como objeto y escenario de su estrategia de confrontación al Estado y en particular al Gobierno Duque. Asuntos que claramente trascienden al nivel nacional y se conectan con la respuesta o "solución" a la problemática del narcotráfico y al logro de la "paz completa" 48 .

Precisamente, en este sentido debe señalarse el reto político vinculado con el desenlace de la coyuntura político nacional, prevista para el 2022, en tanto la posibilidad de encontrar convergencia y propósitos comunes de transformación de las condiciones estructurales, a las que hemos hecho referencia, pero también de la polarización política, va a depender en parte del resultado. Esto además, en tanto el Cali y el Valle aparecen como una de las regiones políticamente más activas y movilizadas y donde el tono y el resultado de la confrontación política serán claves no sólo respecto de los vínculos con la propuesta nacional que reciba el respaldo de la mayoría de los y las colombianas, sino por la conexión y las alternativas de solución con los propios retos y prioridades locales. Se trata de un asunto para nada simple, dada las diferencias de "dinámicas" y tiempos territoriales, pero donde el diálogo local y la movilización social y políticas seguirán siendo fundamental para que, cualquiera sea la opción nacional elegida ${ }^{49}$, se haga posible concertar y darle efectividad a una agenda de transformaciones locales y regionales, que contribuya a remover los factores estructurales invocados o en la base de los conflictos.

Se requiere por tanto una estrategia plural y mancomunada en una perspectiva de gobernanza, en la cual si bien son claves el liderazgo y los programas estatales -tanto del nivel local como regional y nacional- son fundamentales la coordinación y acción concertada de los agentes estatales con y entre los sectores empresarial, educativo, cultural, y las comunidades organizadas; pero igualmente la agencia de todos los actores sociales, políticos e institucionales, que comparten la perspectiva de transformaciones efectivas en el marco de las instituciones del Estado Social de Derecho y para darle efectividad a diversas promesas de la Carta Constitucional de 1991, respecto de una sociedad y un Estado democrático, incluyente y pluralista.

47 Este riesgo viene siendo objeto de la agenda pública y política desde hace varios años y había sido ubicado como asunto prioritario en la agenda del gobierno local desde antes del estallido, señalando la incidencia de complejas estructuras y niveles de criminalidad en la ciudad. Ver Oficina de las Naciones Unidas Contra la Droga y el Delito (UNODC, 2019, p. 31).

48 Se refiere a la necesidad y posibilidad de lograr acuerdos de paz en particular con el ELN y otros grupos insurgentes, lo cual claramente depende no sólo de la disposición del Gobierno sino del real interés en primer lugar del ELN y su dirigencia y de los otros grupos, también dependerá en alto grado del resultado político de las elecciones del 2022; en particular de la coalición y del proyecto político que logre la victoria, y de las políticas respecto del abordaje de la nueva fase del conflicto armado y del narcotráfico.

49 En este momento como alternativas con algún nivel de posicionamiento político aparecen, en la izquierda el Pacto Histórico liderado por Gustavo Petro, quien conforme la mayoría de sondeos, es casi fijo para la segunda vuelta- en el centro-izquierda la Coalición de la Esperanza, en la que participan el Nuevo Liberalismo, un sector del Partido Verde, sectores liberales, el partido Oxígeno Verde y un sector del Polo Democrático; en la centro-derecha la Coalición de la Experiencia, conformada por el partido de la U, sectores de Cambio Radical y del Centro Democrático y otros grupos; en la derecha, el Centro Democrático con la candidatura de Zuluaga y un Outsider: Rodolfo Hernández. 


\section{Referencias bibliográficas}

ACOPI. (2021). Impacto del Paro Nacional 2021. En el sector Mipyme. Recuperado de https://www.acopi.org.co/wpcontent/uploads/2021/07/IMPACTO-DEL-PARO-NACIONAL-2021-EN-EL-SECTOR-MIPYMES-VF.pdf.

Alcaldía de Santiago de Cali. (31 de mayo de 2021). [Decreto 4112.010.20.0304 de 2021]. BO.: 85.

Álvarez-Rodríguez, A. A. (2021). Pistas para entender el 'estallido social' en Cali. Recuperado de https:// www.univalle.edu.co/loque-pasa-en-la-u/pistas-para-entender-el-estallido-social-en-cali.

Auto del juzgado 016 administrativo oral de la ciudad de Cali-Valle del cauca 603/2021, del 11 de junio de 2021.

Centro de Investigación y Documentación Socioeconómica [CIDSE]. (2021). Pensar la resistencia. Cali: Universidad del Valle. Facultad de Ciencias Sociales y Económicas. Recuperado de http://www.cidesco.org.co/wpcontent/uploads/sites/4/2021/06/Libro-La-Resistencia-1.pdf.

Comisión Interamericana de Derechos Humanos [CIDH]. (2021). Observaciones y recomendaciones. Visita de trabajo a Colombia. Recuperado https://www.oas.org/es/cidh/informes/pdfs/ObservacionesVisita_cidh_Colombia_spA.pdf.

Concejo de Santiago de Cali. (10 de noviembre de 2021). [Acuerdo 0509 de 2021]. BO.: 190.

Consejería Presidencial para los Derechos Humanos y Asuntos Internacionales. (2021). Boletín \#30: Derechos Humanos en el marco del Paro $\quad$ Nacional $2021 . \quad$ Recuperado de http://www.derechoshumanos.gov.co/Prensa/2021/Documents/120621-ESP-Infografia-DDHH-Paro-Nacional2021.pdf.

Cruz-Hoyos, S. (13 de junio de 2021). Así fue como el vandalismo quebró a Cali, una ciudad que se levanta de las cenizas. El país, p. A1. Recuperado de https://www.elpais.com.co/cali/asi-fue-como-el-vandalismo-quebro-a-una-ciudad-quese-levanta-de-las-cenizas.html.

Diario Occidente. (10 de diciembre de 2021). Cali lideró la lista más votada de Consejos de Juventud en el país. Diario Occidente. Recuperado de https:/ / occidente.co/cali/cali-lidero-la-lista-mas-votada-de-consejos-de-juventud-en-el-pais/.

El espectador. (3 de junio de 2021). Fedesarrollo estima que el paro habría costado entre 4,8 y \$6 billones en mayo. El espectador. Recuperado de https://www.elespectador.com/economia/fedesarrollo-estima-que-el-paro-habria-costado-entre-48y-6-billones-en-mayo-de-2021/.

El País. (30 de mayo de 2021). Los vecinos del Oeste de Cali que apuestan por la reconciliación. El País. Recuperado de https://www.elpais.com.co/cali/los-vecinos-del-oeste-de-que-apuestan-por-lareconciliacion.html\#: :text=Los\%20vecinos\%20del\%20Oeste\%20de\%20Cali\%20que\%20apuestan\%20por\%20la\%20re conciliaci\%C3\%B3n,Mayo \%2030\%2C\%202021\&text=Son\%20hasta\%20ahora\%2077.,iniciativas\%20de\%20paz\%20del\%20oeste'.

Entelman, R. F. (2005). Teoría de conflictos: hacia un nuevo paradigma. España: Gedisa

Gobernación del Valle de Cauca. (13 de mayo de 2021). [Decreto 1.17.0517 de 2021].

Hernández-Lara, J. (2021). De la marcha hacia el centro al bloqueo en los barrios: las luchas por reconocimiento y oportunidades en Cali durante el Paro Nacional de abril-mayo de 2021. En Centro de Investigación y Documentación Socioeconómica [CIDSE], Pensar la resistencia (pp. 127-150). Cali: Universidad del Valle. Facultad de Ciencias Sociales y Económicas.

Maiese, M., y Lederach, J. (2004). Transformación de conflictos. Recuperado de file:// C:/Users/usuario/Desktop/Transformaci\%C3\%B3n-de-conflictos-Lederach\%20Maiese.pdf.

Ministerio del Interior de Colombia. (28 de mayo de 2021). [Decreto 575 de 2021].

Muñoz-Muñoz, F. A., Herrera-Flores, J., Molina-Rueda, B., y Sánchez-Fernández, S. (2005). Investigación de la paz y los derechos humanos desde Andalucía. España: Editorial Universidad de Granada.

Oficina de las Naciones Unidas Contra la Droga y el Delito [UNODC]. (2019). Informe final del proceso de implementación de la metodología de Auditorías de Seguridad en Santiago de Cali. Documento Técnico de Soporte para la Política Pública de Seguridad y Convivencia de Santiago de Cali. Bogotá: UNODC. Recuperado de https://insightcrime.org/wpcontent/uploads/2021/06/UNODC-ALCALDI\%CC\%81A-DE-CALI.-2019-AUDITORI\%CC\%81A-DESEGURIDAD.pdf.

Oficina del Alto Comisionado de las Naciones Unidas [ACNUDH]. (2021). El Paro Nacional 2021. Lecciones Aprendidas para el ejercicio del derecho de reunión pacífica en Colombia. Recuperado de https://www.colectivodeabogados.org/wpcontent/uploads/2021/12/Colombia_Documento-lecciones-aprendidas-y-observaciones-Paro-Nacional-2021-2.pdf.

Salazar-Trujillo, B. (2021). No salgas de tu barrio: Cali entre el horror y la esperanza. En Centro de Investigación y Documentación Socioeconómica [CIDSE], Pensar la resistencia (pp. 151-165). Cali: Universidad del Valle. Facultad de Ciencias Sociales y Económicas.

Sánchez-Salcedo, J. F. (2021). Reflexiones sobre prácticas y culturas políticas en el paro del 2021. En Centro de Investigación y Documentación Socioeconómica [CIDSE], Pensar la resistencia (pp. 54-66). Cali: Universidad del Valle. Facultad de Ciencias Sociales y Económicas.

Semana.com. (8 de mayo de 2021). Petro le dijo al Comité de Paro que debieron haber frenado las manifestaciones cuando se retiró la reforma tributaria. Semana.com. Recuperado de https:// www.semana.com/nacion/articulo/petro-le-dijo-alcomite-de-paro-que-debieron-haber-frenado-las-manifestaciones-cuando-se-retiro-la-reforma-tributaria/202104/. 


\section{OTROS ARTÍCULOS DE PROSPECTIVA No. 33}

\section{EDITORIAL}

El Paro Nacional en Colombia 2021: explosión social entre dinámicas estructurales y de coyuntura. Relevancia de la acción política y del diálogo en su desarrollo y transformación Adolfo Adrián Álvarez-Rodríguez

\section{ARTÍCULOS}

Organizaciones sociales de pobladores y prácticas de resistencia en contexto de pandemia COVID-19 en Chile

Patricia Castañeda-Meneses

Entre el aislamiento y las brechas digitales: sistematización de experiencia de acompañamiento socioemocional en personas mayores de Temuco, Chile, en tiempos de COVID-19

G. Bernarda Aedo-Neira

Percepción de apoyo social y calidad de vida: la visión de personas mayores chilenas en el contexto de pandemia durante el 2020

Claudia Elena Quiroga-Sanzana

Gabriela Rocío Parra-Monje

Camila Julia Moyano-Sepúlveda

Marco Alejandro Díaz-Bravo

A propósito del qué, cómo y para qué investigar en el campo de la justicia juvenil: La trastienda de un proceso de investigación en Rosario, Argentina Karina De Bella

Proyección social: reflexión sobre una experiencia virtual con adolescentes en época de pandemia en Sucre y Bolivar, Colombia

Claudia Yaneth Martínez-Mina
La intervención social mediante proyectos comunitarios en modalidad virtual en Mérida, México. Una perspectiva desde el Trabajo Social Claudia Isabel Tzec-Puch Amairani Aracelly Ceh-Alvarado Yanet Guadalupe González-Canul

Resignificación de los vínculos Sociales durante la pandemia de COVID-19: narrativas de personas que convergen en Conjuntos residenciales en Bogotá, Colombia

Andrea Marcela Reyes-García

Laura Daniela Molina-Ortiz

La paradoja de la familia doblemente recluida: clínica de una pandemia en Colombia

Norman Darío Moreno-Carmona

Juan José Cleves-Valencia

Ciencias sociales, mundo y pandemia: por un futuro posible

José Miguel Segura-Gutiérrez

Lina Paola Vásquez-Ávila

¿Qué es Trabajo Social? Los primeros vínculos de los estudiantes con la profesión: experiencias de Argentina, Brasil, Paraguay y Uruguay

Silvia Orieta Rivero-Rodríguez

Leonel Del Prado

Nidia Graciela Battilana-Amarilla

Rosilaine Coradini-Guilherme

Intervención profesional de trabajadores sociales de la universidad del Quindio, Colombia. Asuntos epistemológicos, metodológicos y ético-políticos

Ana María Gil-Ríos 
Reflexiones sobre la intervención social en lo rural: experiencias en el Magdalena Medio, Colombia

Claudia Milena Quijano-Mejía

Johana Linares-García

Construcción de rutas de atención integral a la convivencia escolar en Cali, Colombia: sistematización de la experiencia

Leidy Johana Prado-Montaño

Luz Helena López-Rodríguez

Alejandra Gutiérrez-Cárdenas

Experiencia del proceso de intervención familiar de los actores participantes en la Escuela para Familias del ICBF-Jamundí, Colombia Katherin Viviana Silva-Minotta

María José Forero-Izquierdo

Jimena del Pilar Jaramillo-Jaramillo

\section{RESEÑAS DE LIBROS}

Alerta global: políticas, movimientos sociales y futuros en disputa en tiempos de pandemia Jairo Crispín

El neoliberalismo como teología política. Habermas, Foucault, Dardot, Laval y la historia del capitalismo contemporáneo

José Francisco Desentis-Torres

La Reconceptualización del Trabajo Social en Colombia: Análisis histórico-crítico de las décadas de 1960-1970

Juan Pablo Sierra-Tapiro

ARTISTA INVITADO

Juan Camilo González

Prospectiva

\section{PROSPECTIVA}

Revista de Trabajo Social e Intervención Social

No. 33 • ene.-jun. 2022

e-ISSN: 2389-993X • Universidad del Valle 